\title{
A Two Flow Expression Method of Four-Channel Bilateral Control System with Time Delay and Validation of Model-Free Time Delay Compensator
}

\author{
Shoyo Hyodo* Member, Kouhei Ohnishi* Fellow
}

(Manuscript received Oct. 15, 2014, revised Feb. 8, 2015)

\begin{abstract}
Bilateral control is one of the control methods for teleoperation system. The presence of network delays between a master robot and a slave robot makes the design of bilateral control system challengeable. It seriously deteriorates the performance and possibly makes the system unstable. In this paper, to overcome the destabilization from network delay, the model-free time delay compensator is proposed. The proposed compensator does not utilize time delay model and plant model, but the bilateral control system is stabilized. The stability of the proposed control system is shown by using two flow expression method. Two flow expression method models the master system and the slave system of four-channel bilateral control system as single input and single output system. This method makes the analysis for the stability and the performance of the four-channel bilateral control system simple. The validity of the proposed model-free time delay compensator is confirmed by numerical and experimental results.
\end{abstract}

Keywords: haptics, bilateral control, network delay, stability

\section{Introduction}

Recent advances in control theory opened the way towards extension of human manipulation capabilities ${ }^{(1)}$. Benefiting from the advantages of network communication and bilateral control theory, operation over a remote environment (i.e. teleoperation) becames one of the most prominent research topics. Bilateral teleoperation system enables human being to feel a tactile sensation of an environment at remote sight through a master robot and a slave robot. Human beings can manipulate a slave robot in space ${ }^{(2)}$, water, nuclear plants and so on, as if they are in the remote place. Recently, application of this technology is expanded to medical, communication and industry fields ${ }^{(3)}$.

In bilateral control systems, decomposition into two components, i.e. force control system and position control system, is important. Acceleration based four-channel bilateral control is effective to decompose position control and force control of a master robot and a slave robot. The controllers utilize the information of the master/slave position and force. Position and force control are realized in two decomposed virtual modal spaces, i.e. differential mode and common mode ${ }^{(4)}$.

The presence of network delays between a master robot and a slave robot makes the design of bilateral control system challengeable. It seriously deteriorates the performance and possibly makes the system unstable ${ }^{(5)}$. The passivity based approach is very common to stabilize the two-channel bilateral control system ${ }^{(6)}$. This scheme restricts output flows smaller than input flows by using wave variables. Recently,

\footnotetext{
* Department of System Design Engineering, Keio University

3-14-1, Hiyoshi, Kohoku-ku, Yokohama 223-8522, Japan
}

the wave variable scheme has been expanded to four-channel bilateral control system ${ }^{(7)}$. Time delay compensation method is also useful to treat the time delay system without restricting output flows ${ }^{(8)}$. A smith predictor is often utilized to compensate communication delay with a time delay model. But, time delay in network is varying and unpredictable.

A disturbance observer is utilized to deal with time delay systems. The communication disturbance observer (CDOB) has been proposed to compensate time delay effect without a time delay model ${ }^{(9)}$. The effectiveness of time delay compensation by $\mathrm{CDOB}$ to acceleration based four-channel bilateral control system is confirmed experimentally so far. Furthermore, structural approach is also useful to overcome time delay. Acceleration based three-channel control system is confirmed that it has almost same transmission characteristics of force and position information between a master robot and a slave robot compared to $\mathrm{CDOB}^{(10)(11)}$. Three-channel controllers utilize the information of master force and slave position and force.

The performances of CDOB and three-channel control architecture have been confirmed analytically and experimentally. In the point of the stability, it is shown that position control is stabilized. However, force control is not stabilized. Thus, the control system is stable during free motion, but the system can be destabilized when a slave robot contacts with the environment. To stabilize force control in bilateral control system, high frequency domain damping ${ }^{(11)}$ and delayed force feedback method ${ }^{(12)}$ have been proposed. However, these methods need time delay information to construct the control system.

In this paper, model-free time delay compensator is proposed. The proposed control system does not utilized time delay model and plant model, but position control and force 
control of the bilateral control system are stabilized. Moreover, the stability of the proposed control system is verified by the two flow expression of four-channel bilateral control system. In the conventional study, four-channel bilateral control system has been redesigned as two-channel control system by using equilibrium force ${ }^{(13)}$. In this paper, four-channel bilateral control system is modeled as two flow system by using acceleration and transfer functions of the master system and the slave system are derived for analyzing the stability and the performance. This method makes the analysis for the stability and the performance of the four-channel bilateral control system simple. Finally, the validity of the proposed model-free time delay compensator is confirmed by experimental results.

The composition of this paper is described as follows. In Sect. 2, bilateral control based on acceleration control is described. In Sect. 3, two flow expression method of fourchannel bilateral control system is proposed. The master system and the slave system of four-channel bilateral control system are modeled as single input and single output (SISO) system by using this method. In Sect. 4, model-free time delay compensator is proposed. Moreover, the stability and the performance of the proposed control system are verified by two flow expression method. The validity of the proposed model-free time delay compensator is shown by experiment in Sect. 5. Conclusions are described in Sect. 6.

\section{Bilateral Control based on Acceleration Con- trol}

In this section, acceleration based four-channel bilateral control system is explained.

2.1 Disturbance Observer In actual robot control, disturbance force $f^{\text {dis }}$ and external force $f^{e x t}$ as well as reference force $f^{r e f}$ are added to the robot. Disturbance force $f^{d i s}$ includes load force, frictional force and force caused by parametric fluctuation of robot mass $M$ and thrust coefficient $K_{t}$. A disturbance observer (DOB) estimates compensation current $i^{c m p}$ for robust motion control ${ }^{(14)}$. The block diagram of DOB is shown in Fig. 1. Note that the subscript $n$ means nominal value and $g_{d i s}$ is cut-off frequency of low pass filter (LPF).

DOB is also utilized as a reaction force observer (RFOB) for estimation of reaction force ${ }^{(15)}$. While RFOB estimates wider band force information than force sensors, it requires identification of frictional force in advance. In this research, frictional force is not considered, since linear motors which have little friction effect are utilized.

2.2 Four-Channel Bilateral Control System Two robots are considered in bilateral control system, a master robot which is manipulated by human operators and a slave robot which contacts remote environment. Figure 2 shows acceleration based four-channel bilateral control system. DOB and RFOB are implemented in the master robot and the slave robot for robust acceleration control. In Fig. 2, $x_{m}^{r e s}, x_{s}^{r e s}, \hat{f}_{m}^{e x t}$ and $\hat{f}_{s}^{e x t}$ are position response of the master robot, position response of the slave robot, reaction force added to the master robot and reaction force added to the slave robot, respectively. Reaction force added to each robot $\hat{f}_{m}^{e x t}$ and $\hat{f}_{s}^{e x t}$ are estimated using not force sensors but RFOB. $T_{1}$ denotes delay time from the master robot to the slave robot

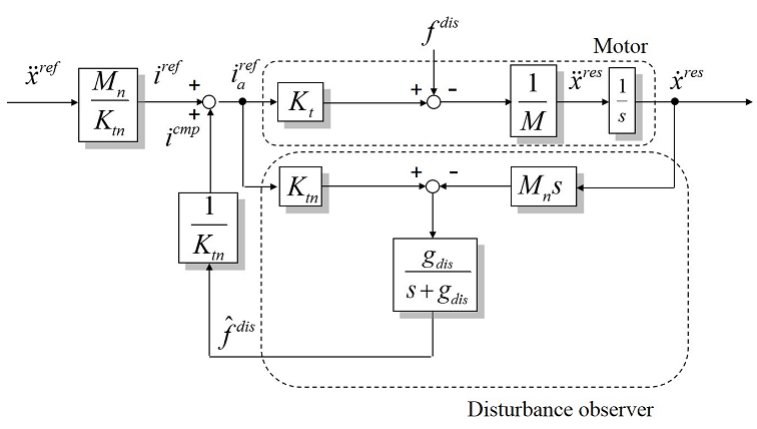

Fig. 1. Disturbance observer

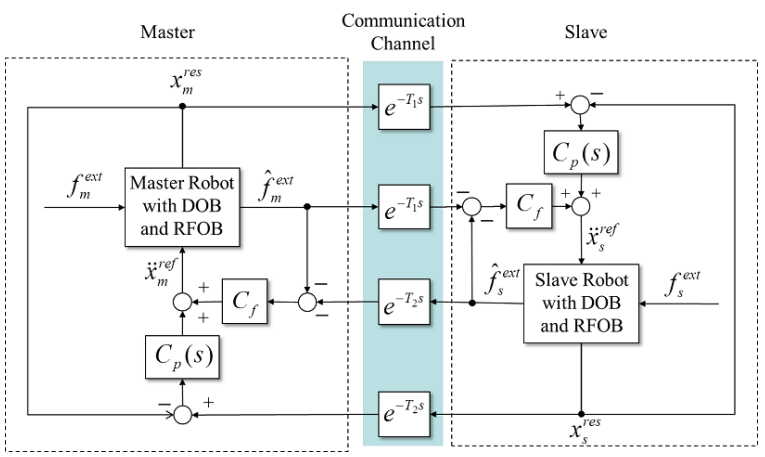

Fig. 2. Acceleration based four-channel bilateral control system

and $T_{2}$ denotes delay time from the slave robot to the master robot. $C_{p}$ is position controller and $C_{f}$ is fore controller.

\section{Two Flow Expression of Four-Channel Bilat- eral Control System}

In four-channel bilateral control system, the master system and the slave system are sending position and force information and receiving those of them. Thus, the master system and the slave system are multi input and multi output (MIMO) system. MIMO system is not easy to analyze the stability and the performance. Therefore, in this section, two flow expression method of four-channel bilateral control system is proposed. By utilizing two flow expression method, the master system and the slave system are modeled as SISO system.

Firstly, acceleration response of four-channel bilateral control system is analyzed. If cut-off frequency of LPF in DOB approaches infinity, the acceleration responses of the master robot and the slave robot in Fig. 2 are expressed as follows.

$$
\begin{aligned}
s^{2} x_{m}^{r e s}= & C_{p}(s)\left(e^{-T_{2} s} x_{s}^{r e s}-x_{m}^{r e s}\right) \\
& -C_{f}\left(e^{-T_{2} s} \hat{f}_{s}^{e x t}+\hat{f}_{m}^{e x t}\right) \\
s^{2} x_{s}^{r e s}= & C_{p}(s)\left(e^{-T_{1} s} x_{m}^{r e s}-x_{s}^{r e s}\right) \\
& -C_{f}\left(e^{-T_{1} s} \hat{f}_{m}^{e x t}+\hat{f}_{s}^{e x t}\right)
\end{aligned}
$$

Here, DOB works ideally and disturbance force including parameter fluctuation is suppressed completely, i.e. $x_{m}^{\text {res }}=$ $\ddot{x}_{m}^{r e f} / s^{2}$ and $x_{s}^{r e s}=\ddot{x}_{s}^{r e f} / s^{2}$. Thus, Eqs. (1)-(2) do not include dynamics such as mass variable. Equations (1)-(2) are not realized in high-frequency area above cut-off frequency of LPF in DOB $\left(g_{d i s}\right)$. In usual case, $g_{d i s}$ can be set large enough, so this assumption is negligible in practical use. 


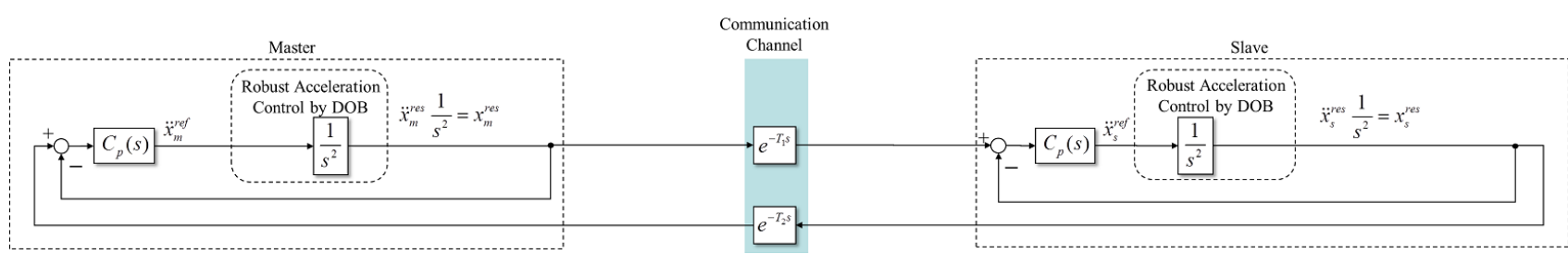

(a) Position control of four-channel bilateral control system.

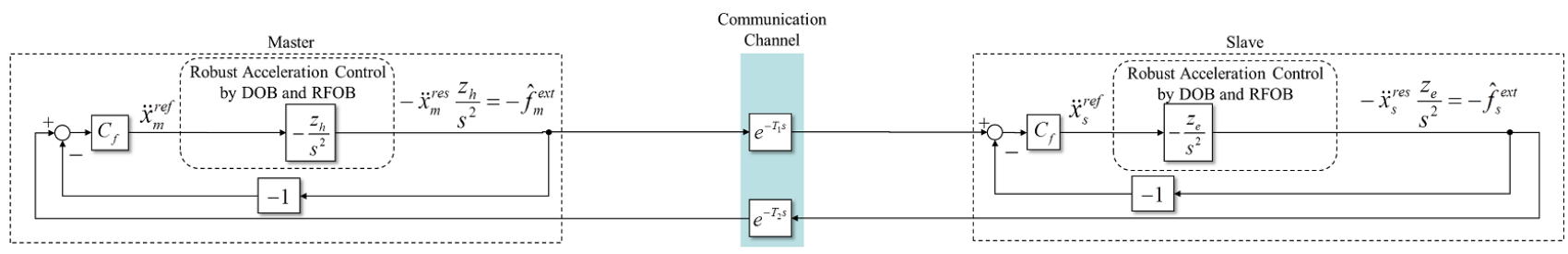

(b) Force control of four-channel bilateral control system.

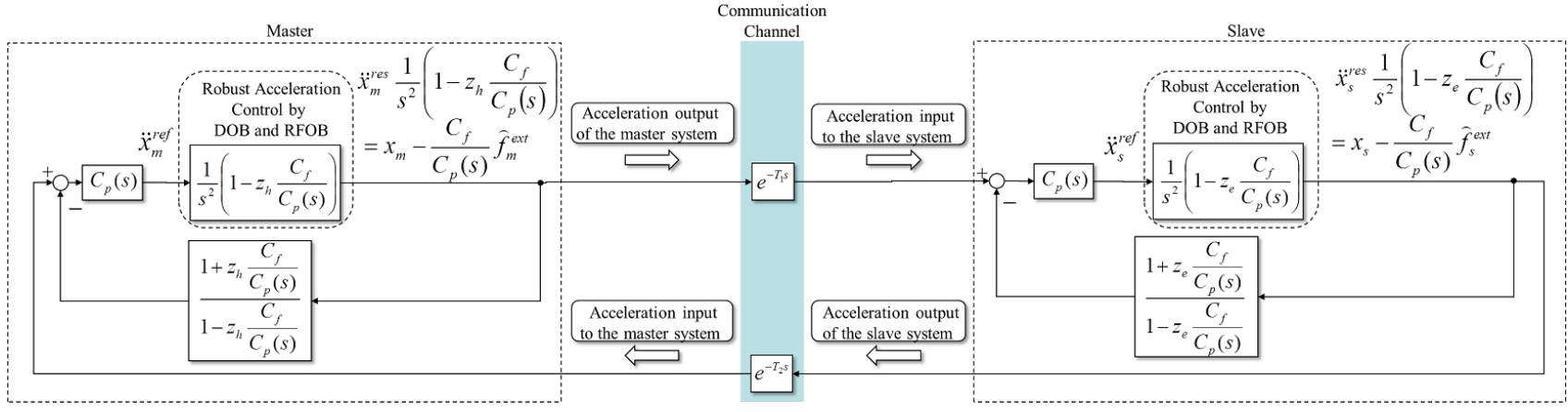

(c) Position and force control of four-channel bilateral system.

Fig. 3. Two flow expression of acceleration based four-channel bilateral control system

Then, position control and force control of the bilateral control system are decomposed as follows.

$$
\begin{aligned}
s^{2} x_{m}^{r e s, p} & =C_{p}(s)\left(e^{-T_{2} s} x_{s}^{r e s}-x_{m}^{r e s}\right) \cdots \\
s^{2} x_{s}^{r e s, p} & =C_{p}(s)\left(e^{-T_{1} s} x_{m}^{r e s}-x_{s}^{r e s}\right) \cdots \\
s^{2} x_{m}^{r e s, f} & =-C_{f}\left(e^{-T_{2} s} \hat{f}_{s}^{e x t}+\hat{f}_{m}^{e x t}\right) \cdots \\
s^{2} x_{s}^{r e s, f} & =-C_{f}\left(e^{-T_{1} s} \hat{f}_{m}^{e x t}-\hat{f}_{s}^{e x t}\right) \cdots
\end{aligned}
$$

Here, $s^{2} x_{m}^{r e s, p}$ or $s^{2} x_{s}^{r e s, p}$ are acceleration response of position control, $s^{2} x_{m}^{r e s, f}$ or $s^{2} x_{s}^{r e s, f}$ are acceleration response of force control.

From Eqs. (3) and (4), the block diagram for position control of the bilateral control system is expressed as Fig. 3(a). From Eqs. (5) and (6), the block diagram for force control of the bilateral control system is expressed as Fig. 3(b). In Fig. 3(b), RFOB works ideally and wideband force sensation is realized, i.e. $\hat{f}_{m}^{r e s}=x_{m}^{r e s} z_{h}$ and $\hat{f}_{s}^{r e s}=x_{s}^{r e s} z_{e} . z_{h}$ means human impedance and $z_{e}$ means environmental impedance. Fig. 3(b) is not realized in high-frequency area above cut-off frequency of LPF in RFOB $\left(g_{r f o b}\right)$. In usual case, $g_{r f o b}$ can be set large enough, so this assumption is negligible in practical use.

Finally, by summing Fig. 3(a) and (b), Fig. 3(c) is derived. As shown in Fig. 3(c), the master system and the slave system are modeled as SISO system. Input and output of each system is acceleration information as shown in Fig. 3(c). Acceleration $\ddot{x}_{m}^{r e s}$ or $\ddot{x}_{s}^{r e s}$ work as an agent to connect both control systems. From the block diagrams of the master system and the slave system in Fig. 3(c), transfer functions of each system are derived in Sect. 4.2.

\section{Validation of Model-Free Time Delay Com- pensator}

In this section, model-free time delay compensator is proposed. Moreover, the stability and the performance of the proposed control system are shown by using two flow expression method.

4.1 Model-Free Time Delay Compensator Modelfree time delay compensator is inspired from the idea of CDOB. Figure 4 shows a feedback control system with CDOB and Smith predictor. Figure 4(a) shows a feedback control system with Smith predictor and Fig. 4(b) shows a feedback control system with CDOB. In the figure, $G(s)$ is a controlled plant, $R(s)$ and $Y(s)$ are an input and an output, respectively. $T_{3}$ denotes delay time from local system to remote system. $T_{4}$ denotes delay time from remote system to local system. $\hat{G}(s)$ is a plant model and $\hat{T}_{5}$ is a round trip delay time $\left(T_{5}=T_{3}+T_{4}\right)$ model. From Fig. 4(a), Smith predictor stabilizes the feedback control system by utilizing time delay model $\left(\hat{T}_{5}\right)$ and a plant model $(\hat{G}(s))$. On the other hand, time delay compensation by CDOB which is shown in Fig. 4(b) only utilizes plant model $(\hat{G}(s))^{(16)}$. Here, Fig. 4(c) shows the equivalent transformation of Fig. 4(b). It is shown that CODB works same performance with Smith predictor without a round trip delay time model. This is because that the CDOB calculates compensation value 'comp' by the differentiation of 'comp1' and 'comp2'. This is the biggest advantage in $\mathrm{CDOB}$ approach.

The proposed model-free time delay compensator utilizes the differentiation of the signal before and after going through 
the network as the compensation values like CDOB approach. Examples of the differentiation signals in fourchannel bilateral control system are $x_{m}^{r e s}-x_{m}^{r e s} e^{-T s}$ or $\hat{f}_{m}^{\text {ext }}-$ $\hat{f}_{m}^{e x t} e^{-T s}$. Here, $T$ is a round trip delay time $\left(T=T_{1}+T_{2}\right)$ in Fig. 2. These signals become 0 if the phase lag is around 0 degrees and $2 x_{m}^{\text {res }}$ or $2 \hat{f}_{m}^{\text {ext }}$ if the phase lag becomes around

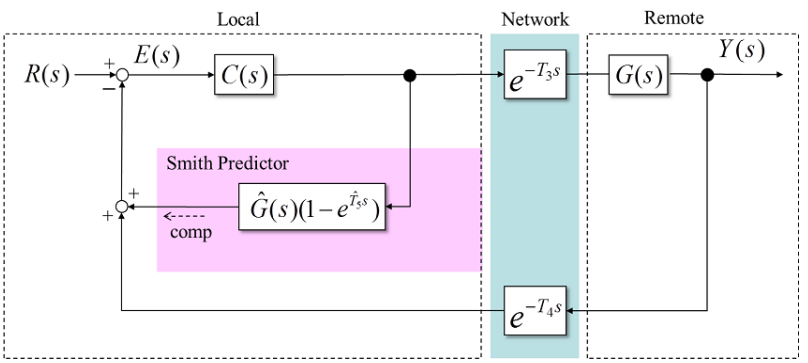

(a) Time delay compensation by Smith predictor.

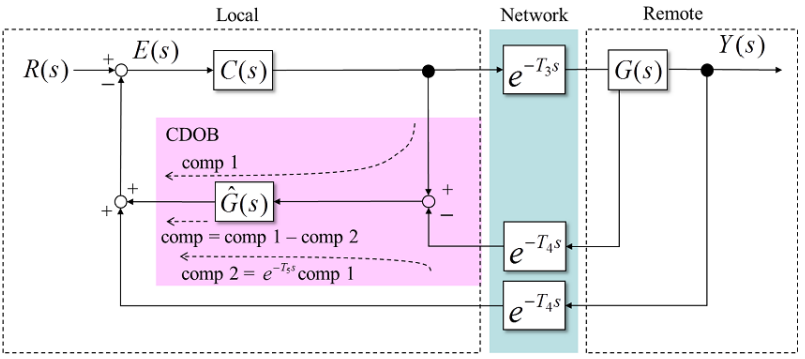

(b) Time delay compensation by CDOB.

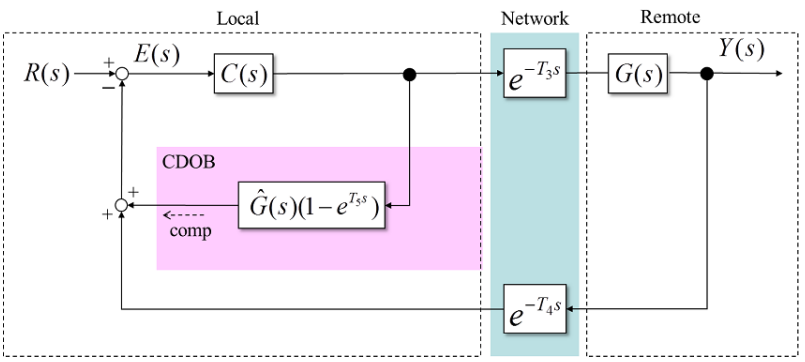

(c) Equivalent transformation of (b)

Fig. 4. Time delay compensation in feedback control system
180 degrees. Thus, these signals are valid to prioritize the information in the master side control system. Then, the acceleration references of the bilateral control system with modelfree time delay compensator are designed as follows.

$$
\begin{aligned}
s^{2} x_{m}^{r e f}= & C_{p}(s)\left(e^{-T_{2} s} x_{s}^{r e s}-x_{m}^{r e s}\right) \\
& -C_{f}\left(e^{-T_{2} s} \hat{f}_{s}^{e x t}+\hat{f}_{m}^{e x t}\right) \\
& +C_{p}(s)\left(e^{-\left(T_{1}+T_{2}\right) s} x_{m}^{r e s}-x_{m}^{r e s}\right) \\
& +C_{f}\left(e^{-\left(T_{1}+T_{2}\right) s} \hat{f}_{m}^{r e s}-\hat{f}_{m}^{r e s}\right) \\
s^{2} x_{s}^{r e f}= & C_{p}(s)\left(e^{-T_{1} s} x_{m}^{r e s}-x_{s}^{r e s}\right) \\
& -C_{f}\left(e^{-T_{1} s} \hat{f}_{m}^{e x t}+\hat{f}_{s}^{e x t}\right)
\end{aligned}
$$

In Eq. (7), third term and fourth term are corresponding to the compensation values. In the case that the phase lag is around 0 degrees, these signals become 0 . Then, the acceleration reference of the master system becomes as Eq. (9).

$$
\begin{aligned}
s^{2} x_{m}^{r e f}= & C_{p}(s)\left(e^{-T_{2} s} x_{s}^{r e s}-x_{m}^{r e s}\right) \\
& -C_{f}\left(e^{-T_{2} s} \hat{f}_{s}^{e x t}+\hat{f}_{m}^{e x t}\right)
\end{aligned}
$$

This is same as the acceleration reference of four-channel bilateral control system for the master system. In the case that the phase lag is around 180 degrees, compensation values become $-2 x_{m}^{\text {res }}$ or $-2 \hat{f}_{m}^{e x t}$. Then, by the compensation values, scaling are added in the master control system as Eq. (10).

$$
\begin{aligned}
s^{2} x_{m}^{r e f}= & C_{p}(s)\left(e^{-T_{2} s} x_{s}^{r e s}-3 x_{m}^{r e s}\right) \\
& -C_{f}\left(e^{-T_{2} s} \hat{f}_{s}^{e x t}+3 \hat{f}_{m}^{e x t}\right)
\end{aligned}
$$

This means that the master system utilizes more information of own system compared with the slave system. This is valid to reduce the time delay effect since general bilateral control systems with time delay can become unstable when the phase lag becomes around 180 degrees.

By utilizing Eqs. (7) and (8), the bilateral control system with model-free time delay compensator is implemented as Fig. 5. Compensation value for position control and force control of the bilateral control system are calculated in

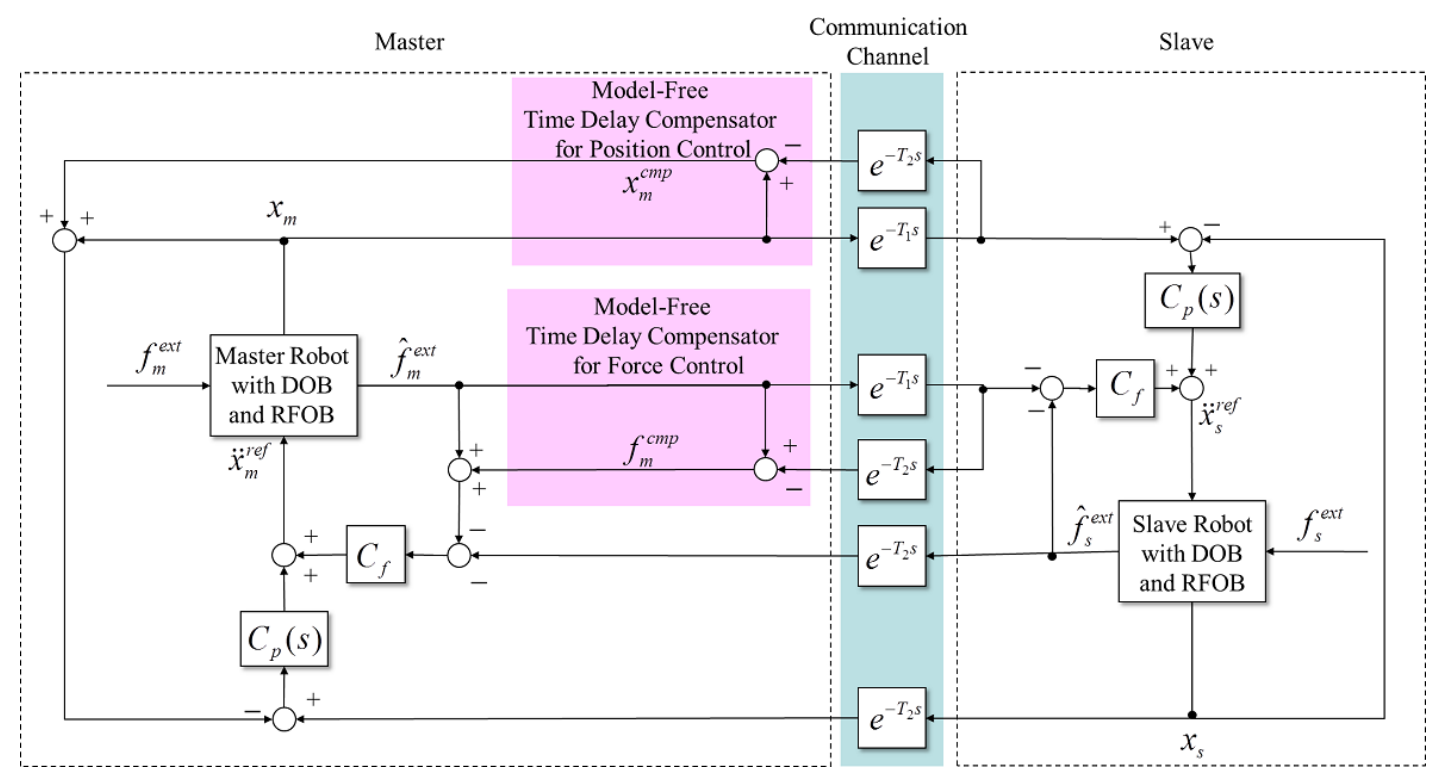

Fig. 5. Bilateral control system with model-free time delay compensator 


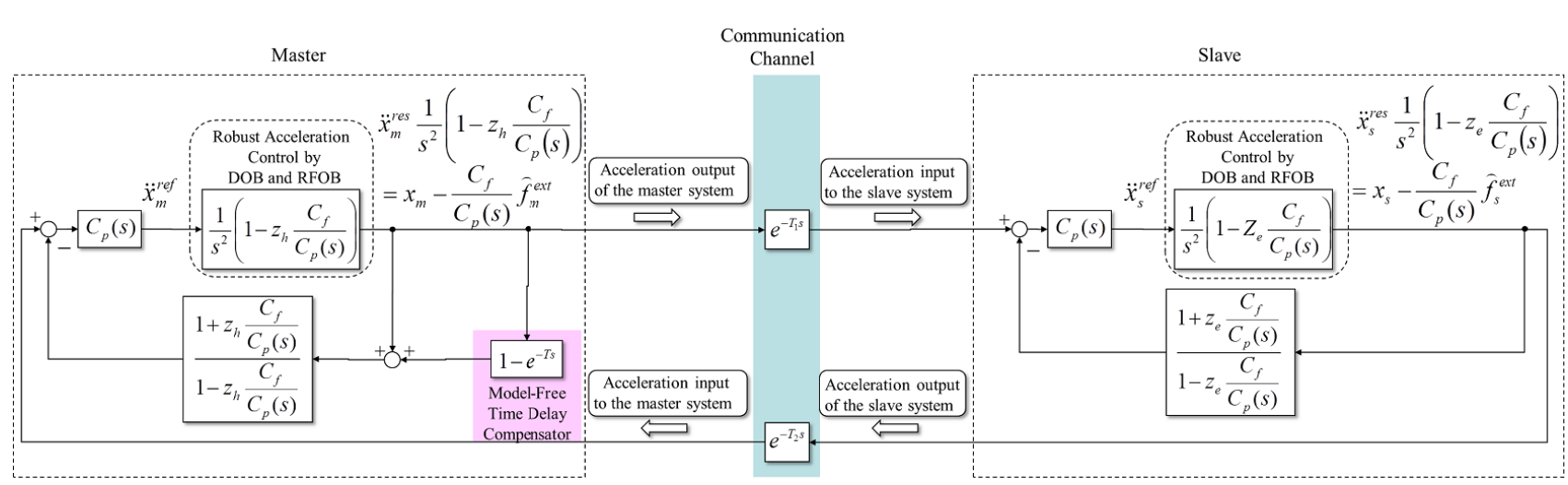

Fig. 6. Two flow expression of bilateral control system with model-free time delay compensator

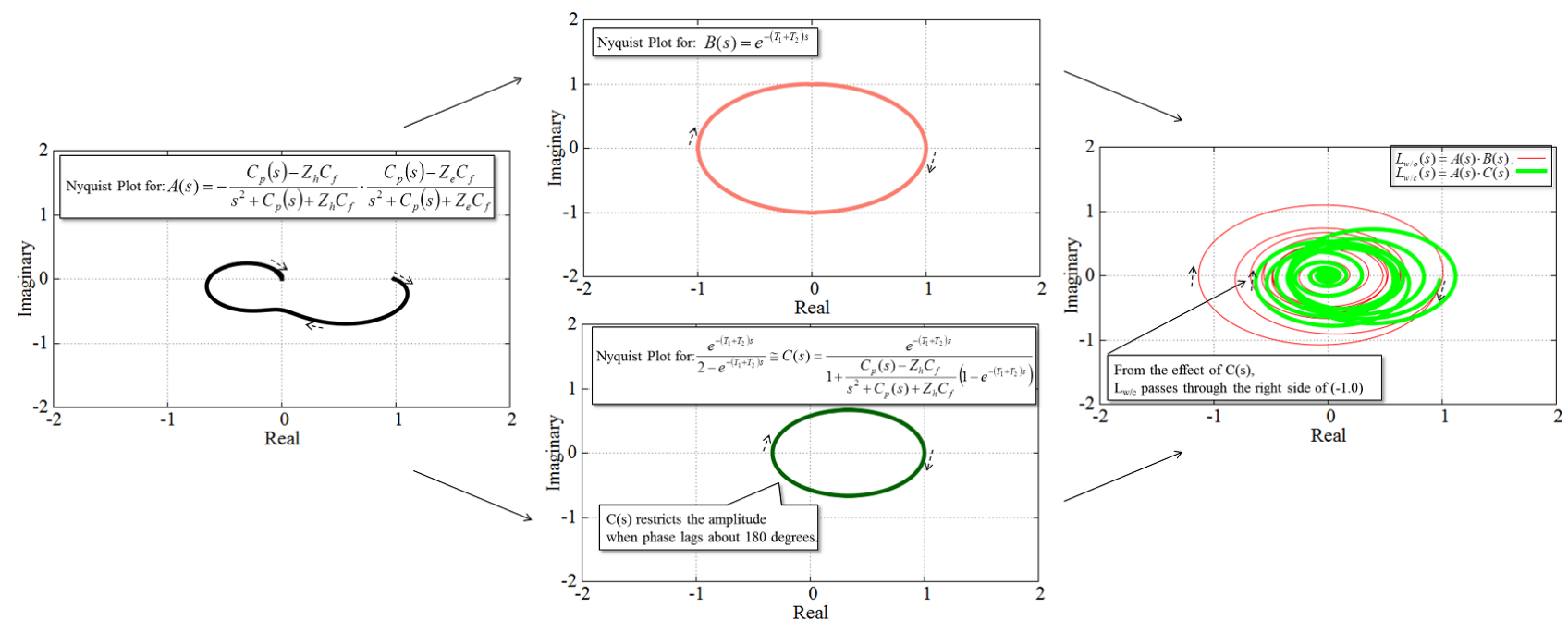

Fig. 7. Nyquist plot for bilateral control system without and with model-free time delay compensator

$$
\begin{aligned}
& L_{w / o}(s)=-\underbrace{\frac{C_{p}(s)-Z_{h} C_{f}}{s^{2}+C_{p}(s)+Z_{h} C_{f}}}_{\text {master system }} \cdot \underbrace{\frac{C_{p}(s)-Z_{e} C_{f}}{s^{2}+C_{p}(s)+Z_{e} C_{f}}}_{\text {slave system }} \cdot \underbrace{e^{-\left(T_{1}+T_{2}\right) s}}_{\text {communication channel }} \\
& L_{w / c}(s)=-\underbrace{\frac{C_{p}(s)-Z_{h} C_{f}}{s^{2}+C_{p}(s)+Z_{h} C_{f}}} \cdot \frac{1}{1+\frac{C_{p}(s)-Z_{h} C_{f}}{s^{2}+C_{p}(s)+Z_{h} C_{f}}\left(1-e^{\left.-T_{2} s\right)}\right.} \cdot \underbrace{\frac{C_{p}(s)-Z_{e} C_{f}}{s^{2}+C_{p}(s)+Z_{e} C_{f}}}_{\text {slave system }} \cdot \underbrace{e^{-\left(T_{1}+T_{2}\right) s}}_{\text {communication channel }} \\
& =-\underbrace{\frac{C_{p}(s)-Z_{h} C_{f}}{s^{2}+C_{p}(s)+Z_{h} C_{f}}}_{\text {master system }} \cdot \underbrace{\frac{C_{p}(s)-Z_{e} C_{f}}{s^{2}+C_{p}(s)+Z_{e} C_{f}}}_{\text {slave syster }} \cdot \underbrace{\frac{C^{-\left(T_{1}+T_{2}\right) s}}{1+\frac{C_{p}(s)-Z_{h} C_{f}}{s^{2}+C_{p}(s)+Z_{h} C_{f}}\left(1-e^{\left.-T_{2} s\right)}\right.}}_{\text {communication channel with compensator }}
\end{aligned}
$$

hatched area of Fig. 5. Here, compensation values are calculated by the differentiation of the signal before and after going through the network. Thus, time delay model and plant model are not utilized to calculate compensation values. So, it is called model-free time delay compensator.

4.2 Stability In this subsection, the stability of the bilateral control system with model-free time delay compensator is discussed by two flow expression method. Figure 6 shows the block diagram of the bilateral control system with model-free time delay compensator in two flow expression. Compared with Fig. 3(c), model-free time delay compensator is added in Fig. 6.

From Fig. 3(c) and Fig. 6, open-loop transfer function for the bilateral control system without model-free time delay compensator $\left(L_{w / o}\right)$ and those for the bilateral control system with model-free time delay compensator $\left(L_{w / c}\right)$ are calculated as Eqs. (11) and (12). Then, these open-loop transfer function are decomposed as (13) to (17) for analyzing the stability.

$$
\begin{aligned}
& L_{w / o}(s)=A(s) \cdot B(s) \\
& L_{w / c}(s)=A(s) \cdot C(s) \\
& A(s)=-\frac{C_{p}(s)-Z_{h} C_{f}}{s^{2}+C_{p}(s)+Z_{h} C_{f}} \cdot \frac{C_{p}(s)-Z_{e} C_{f}}{s^{2}+C_{p}(s)+Z_{e} C_{f}} \\
& \text {................ } \\
& B(s)=e^{-\left(T_{1}+T_{2}\right) s} .
\end{aligned}
$$



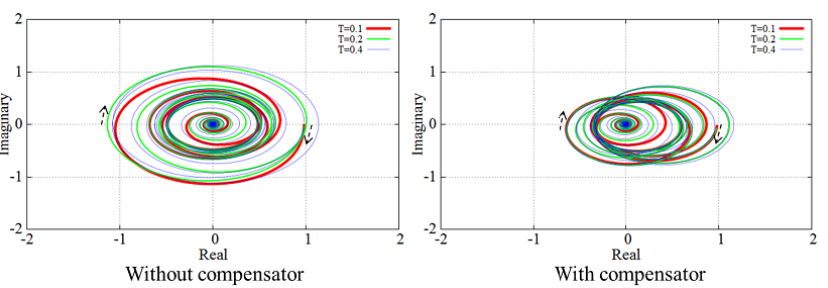

(a) Effect of delay time.
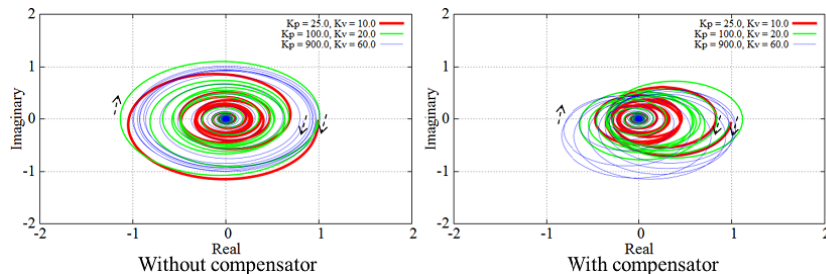

(c) Effect of $\mathrm{Cp}(\mathrm{s})$.
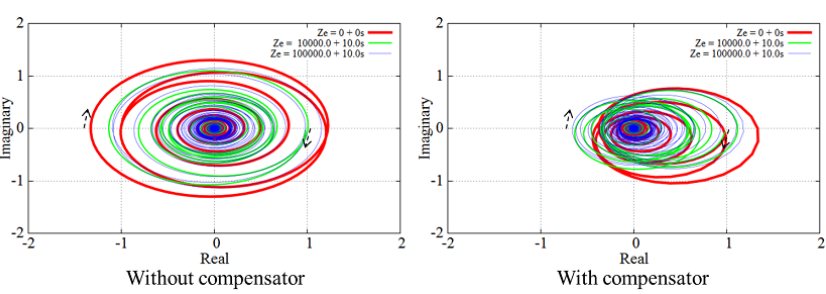

(b) Effect of environmental impedance.
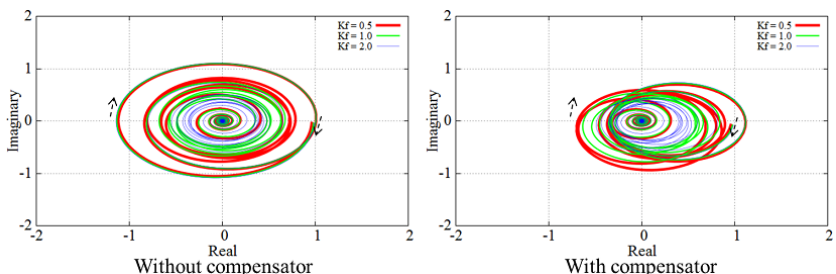

(d) Effect of Cf.

Fig. 8. Nyquist plot for parametric study of stability

Table 1. Parameters for analysis and experiment

\begin{tabular}{l|c||c}
\hline \hline Position feedback gain & $K_{P}$ & 100 \\
Velocity feedback gain & $K_{V}$ & 20 \\
Force feedback gain & $K_{f}$ & 1.0 \\
Impedance of human(for analysis) & $z_{h}$ & $0+0 \mathrm{~s}$ \\
Impedance of environment(for analysis) & $z_{e}$ & $10000+10 \mathrm{~s}$ \\
Nominal mass & $M_{n}$ & $0.5(\mathrm{~kg})$ \\
Thrust coefficient & $K_{t n}$ & $32.5(\mathrm{~N} / \mathrm{A})$ \\
One way delay time & $T$ & $0.2(\mathrm{sec})$ \\
\hline
\end{tabular}

$$
C(s)=\frac{e^{-\left(T_{1}+T_{2}\right) s}}{1+\frac{C_{p}(s)+Z_{h} C_{f}}{s^{2}+C_{p}(s)-Z_{h} C_{f}}\left(1-e^{-\left(T_{1}+T_{2}\right) s}\right)}
$$

Here, $A(s)$ is corresponding to the transfer function of the master system and the slave system with no time delay compensator. $B(s)$ is showing time delay from network communication. $C(s)$ is corresponding to time delay from network communication with model-free time delay compensator.

Figure 7 shows the nyquist plot of those equations. Table 1 shows the parameter for the nyquist plot. Here, impedance of human operator $\left(z_{h}\right)$ is settled as $0+0 s$ to see the stability in safe side. This is because that the control system can be stabilized from the damping effect of the human impedance. From Fig. 7, it is shown that time delay effect $B(s)$ shifts the phase of $A(s)$ that the nyquist plot of $L_{w / o}(s)$ passes through the left side of $(-1,0)$. On the other hand, the time delay effect with model-free time delay compensator $C(s)$ restricts the amplitude of the $A(s)$ when the phase lags about 180 degrees. Thus, the nyquist plot of $L_{w / c}(s)$ passes through the right side of $(-1,0)$. Therefore, the bilateral control system is stabilized when the model-free time delay compensator is added. As shown in Eq. (12), there does not include dynamics such as mass variable in open loop transfer function of $L_{w / c}(s)$. So, the model-free time delay compensator works even if the size of the master system and the slave system are different.

For analyzing the stability in more general case, parametric study for the nyquist has been conducted. Figure 8 shows the parametric study results. In Fig. 8, time delay, impedance of environment, position control gain and force control gain

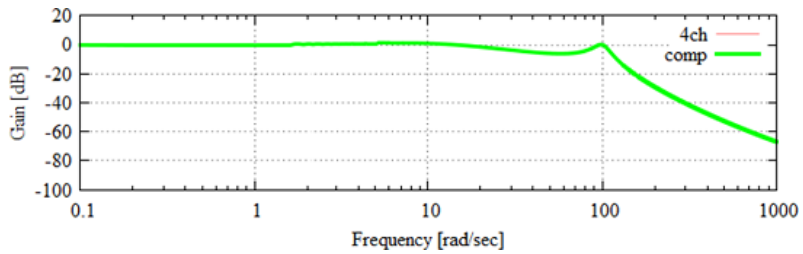

(a) $\mathrm{T}=0 \mathrm{sec}$.

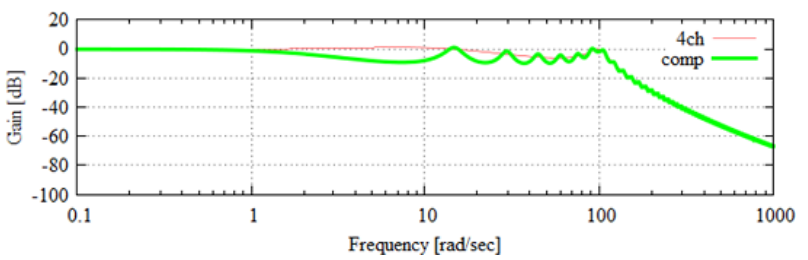

(b) $\mathrm{T}=0.2 \mathrm{sec}$.

Fig. 9. Gain characteristics of open loop transfer function

are parameterized. From Fig. 8, it is shown that the bilateral control system with model-free time delay compensator is always stable. Figure 8(a) shows that the bilateral control system without compensator is not stable in all cases of time delay. From Fig. 8(b), the stability of the bilateral control system without compensator becomes worse when the environmental impedance becomes small. Figure 8(c) shows that the stability of the bilateral control system without compensator is improved by increasing position feedback gain. Figure 8(d) shows that the bilateral control system without compensator is not stable in all cases of force feedback gain. Thus, the model-free time delay compensator is valid to stabilize the bilateral control system with time delay.

4.3 Performance In this subsection, the performance of the bilateral control system with model-free time delay compensator is analyzed by using two flow expression method.

Figure 9 shows the gain characteristics of open loop transfer function. Open loop transfer functions are derived from Eqs. (11) and (12). Figure 9(a) shows those with no delay time $(T=0)$ and Fig. 9(b) shows those with $0.2 \mathrm{sec}$ one way delay time $(T=0.2 s)$.

As shown in Fig. 9(a), gain characteristics are same in bilateral control system without and with model-free time delay 
compensator when there is no time delay. Figure 9(b) shows that gain characteristics are restricted in bilateral control system with model-free time delay compensator when there is $0.2 \mathrm{~s}$ one way time delay. Therefore, as shown in Sect. 4.2, model-free time delay compensator works to restrict performance to stabilize the control system. This restriction works automatically depending on network delay time and does not need time delay model in control system.

\section{Experiment}

In this section, experimental results are shown to confirm the validity of the proposed method.

5.1 Experimental System Figure 10 shows the experimental system. The master and the slave system consist of 1-DOF robots. The robot consists of linear motor and encoder. Human operator manipulates the master side linear motor, and the slave side linear motor contacts environment. Resolution of encoders is $0.1 \mu \mathrm{m}$. Network delay time is created by network emulator. In the experiment, one way delay

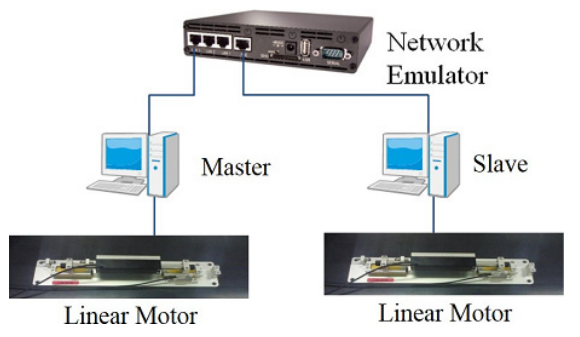

Fig. 10. Experimental system

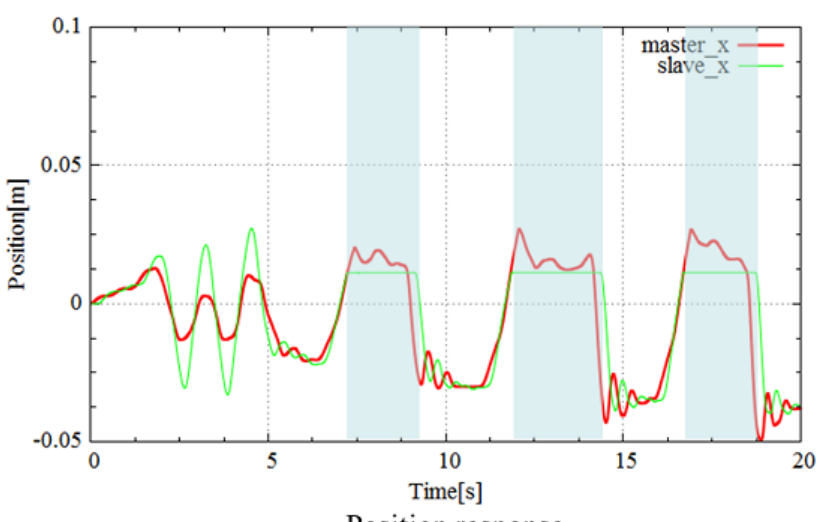

Position response

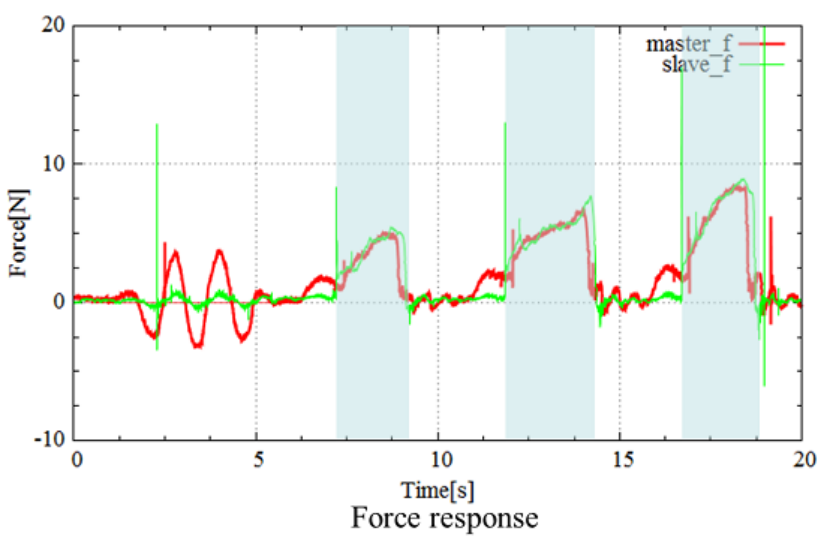

(a) Without Compensator. time is set as $0.2 \mathrm{~s}$. Control parameters used in the experiments are shown in Table 1.

5.2 Experimental Results Figure 11 shows the experimental results. The force response of the master robot is plotted reverse value to compare with the force response of the slave robot. Hatched area is showing that slave robot contacts environment. Figure 11(a) shows the experimental results of without model-free time delay compensator, Fig. 11(b) shows those with model-free time delay compensator.

In the case of without model-free time delay compensator, position response is vibrating during contact with environment. On the other hand, in the case of with model-free time delay compensator, there is no vibration during contact with environment. Therefore, it is shown that model-free time delay compensator is valid to stabilize the bilateral control system with time delay.

\section{Conclusion}

In this paper, the model-free time delay compensator has been proposed to overcome the destabilization from network delay. The proposed compensator does not utilize time delay model and plant model, but position control and force control of the bilateral control system are stabilized. The stability of the proposed control system has been shown by using two flow expression method. Two flow expression method models the master system and the slave system of four-channel bilateral control system as SISO system. This method makes the analysis for the stability and the performance of the fourchannel bilateral control system simple. The validity of the
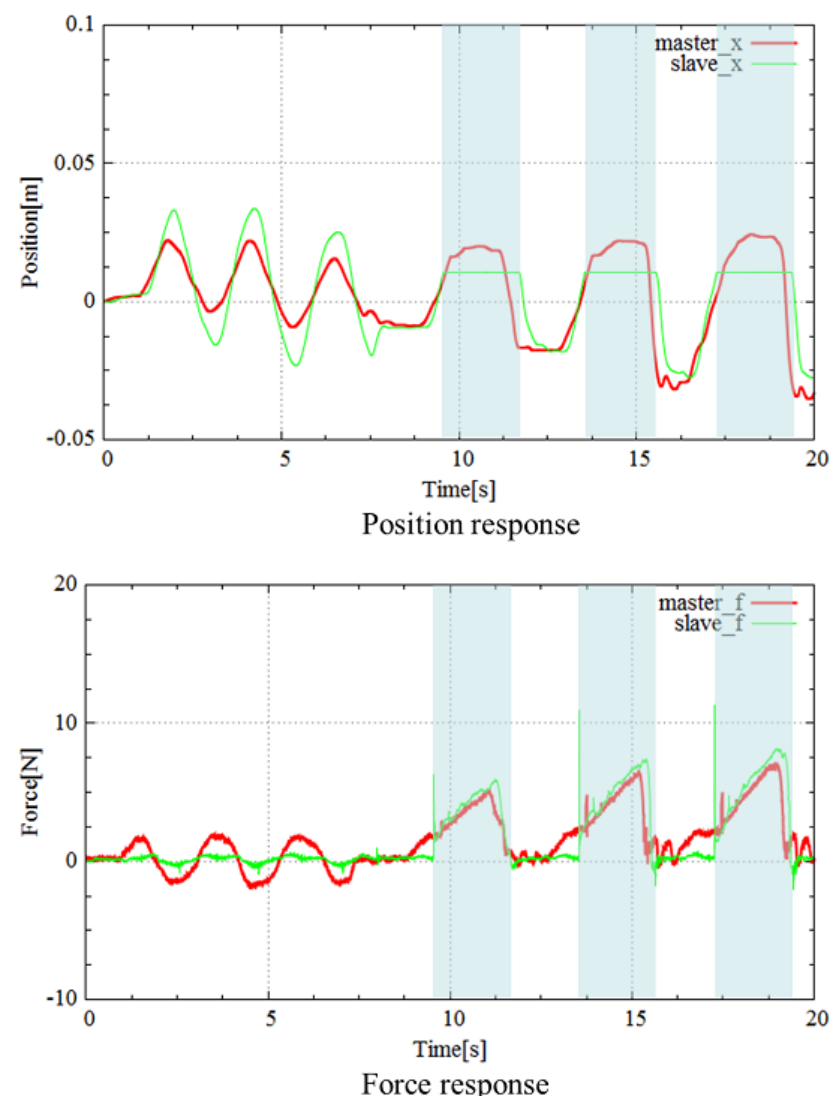

(b) With Compensator.

Fig. 11. Experimental results 
proposed model-free time delay compensator has been confirmed by numerical and experimental results.

\section{Acknowledgment}

This research was supported in part by the Ministry of Education, Culture, Sports, Science and Technology of Japan under Grant-in-Aid for Scientific Research (S), 25220903, 2013.

\section{References}

( 1 ) P.F. Hokayem and M.W. Spong: "Bilateral Teleoperation; An historical survey”, Automatica, Vol.42, No.12, pp.2035-2057 (2006)

(2) T. Imaida, Y. Yokokohji, T. Doi, M. Oda, and T. Yoshikawa: "Ground-Space Bilateral Teloperation of ETS-VII Robot Arm by Direct Bilateral Coupling Under 7-s Time Delay Condition", IEEE Trans. on Robotics and Automation, Vol.20, No.3, pp.499-511 (2004)

( 3 ) K. Ohnishi, S. Katsura, and T. Shimono: "Robot as Physical Agent", Proc. of the IEEE International Conf. on Industrial Technology, Keynote Lecture - I (2006)

( 4 ) Y. Matsumoto, S. Katsura, and K. Ohnishi: "An Analysis and Design of Bilateral Control Based on Disturbance Observer", Proc. of the IEEE Int. Conf. on Industrial Technology, pp.802-807 (2003)

( 5 ) R.J. Anderson and M.W. Spong: "Bilateral Control of Teleoperators with Time Delay”, IEEE Trans. on Automatic Control, Vol.34, No.5, pp.494-501 (1989)

( 6 ) G. Niemeyer and J.J.E. Slotine: "Stable Adaptive Teleoperation", IEEE Journal of Oceanic Engineering, Vol.16, No.1, pp.152-162 (1991)

( 7 ) A. Aziminejad, M. Tavakoli, P.V. Patel, and M. Moallem: "Transparent Time-Delayed Bilateral Teleoperation Using Wave Variables", IEEE Trans. on Control Systems Technology, Vol.16, No.3, pp.548-555 (2008)

( 8 ) J.P. Richard: "Time-delay systems; an overview of some recent advances and open problems", Automatica, Vol.39, No.10, pp.1667-1694 (2003)

( 9 ) K. Natori, T. Tsuji, and K. Ohnishi: "Time-Delay Compensation by Communication Disturbance Observer for Bilateral Tleoperation Under TimeVarying Delay", IEEE Trans. on Industrial Electronics, Vol.55, No.5, pp.2152-2168 (2008)

(10) R. Kubo, N. Iiyama, K. Natori, K. Ohnishi, and H. Furukawa: "Performance Analysis of a Three-Channel Control Architecture for Bilateral Teleoperation with Time Delay", IEEJ Trans. IA, Vol.127, No.12, pp.1224-1230 (2007)

(11) A. Suzuki and K. Ohnishi: "Frequency-Domain Damping Design for TimeDelayed Bilateral Teleoperation System Based on Modal Space Analysis", IEEE Trans. on Industrial Electronics, Vol.60, No.1, pp.177-190 (2013)
(12) S. Hyodo and K. Ohnishi: "A Structure of Bilateral Control System with Time Delay Considering Improvement of Stability", Proc. of the IEEE Int. Conf. on Advanced Motion Control, pp.284-289 (2014)

(13) T. Nozaki, S. Shimizu, T. Mizoguchi, and K. Ohnishi: "Interpretation of Acceleration-Based Bilateral Control Standing on Impedance Control and Its Application to Reduction of Haptic Data", Papers of the IEEJ Technical Meeting on Industrial Instrumentation and Control, Tokyo, Japan, Vol.IIC14, pp.69-74 (2014)

(14) K. Ohnishi, M. Shibata, and T. Murakami: "Motion Control for Advanced Mechatronics", IEEE/ASME Trans. on Mechatronics, Vol.1, No.1, pp.56-67 (1996)

(15) S. Katsura, Y. Matsumoto, and K. Ohnishi: "Analysis and Experimental Validation of Force Bandwidth for Force Control", IEEE Trans. on Industrial Electronics, Vol.53, No.3, pp.922-928 (2006)

(16) S. Hyodo and K. Ohnishi: "A Structure of Time Delayed Control System with Communication Disturbance Observer Considering Unstable Network", Papers of the IEEJ Technical Meeting on Industrial Instrumentation and Control, Tokyo, Japan, Vol.IIC-14, pp.81-86 (2014) (in Japanese)

Shoyo Hyodo (Member) received the B.E. degree in system design en-

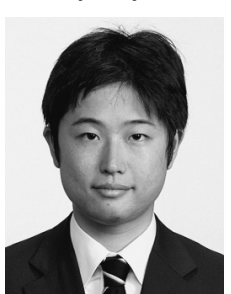
gineering and the M.E. degree in integrated design engineering from Keio University, Yokohama, Japan, in 2006 and 2008, respectively. He is currently with the space system division of Mitsubishi Heavy Industries. From 2013, he starts to make a research toward the Ph.D. degree in Keio University. His research interests include bilateral control over network.

Kouhei Ohnishi

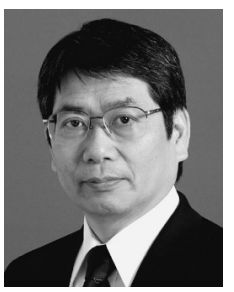

(Fellow) received the B.E., M.E., and Ph.D. degrees in electrical engineering from The University of Tokyo, Tokyo, Japan, in 1975, 1977, and 1980, respectively. Since 1980, he has been with Keio University, Yokohama, Japan and is now a Professor of Department of System Design Engineering. His research interests include motion control,robotics and haptics. 